\title{
Supporting Information for Electronic Structure and Transport in Graphene Nanoribbon Heterojunctions under Uniaxial Strain: Implications for Flexible Electronics
}

\author{
Amir Taqieddin and N. R. Aluru* \\ Department of Mechanical Science and Engineering \\ University of Illinois at Urbana-Champaign, Urbana, Illinois 61801, USA \\ * Corresponding Author, e-mail: aluru@illinois.edu, web: http://aluru.mechse.illinois.edu/
}

This Supporting Information file includes sixteen figures. 


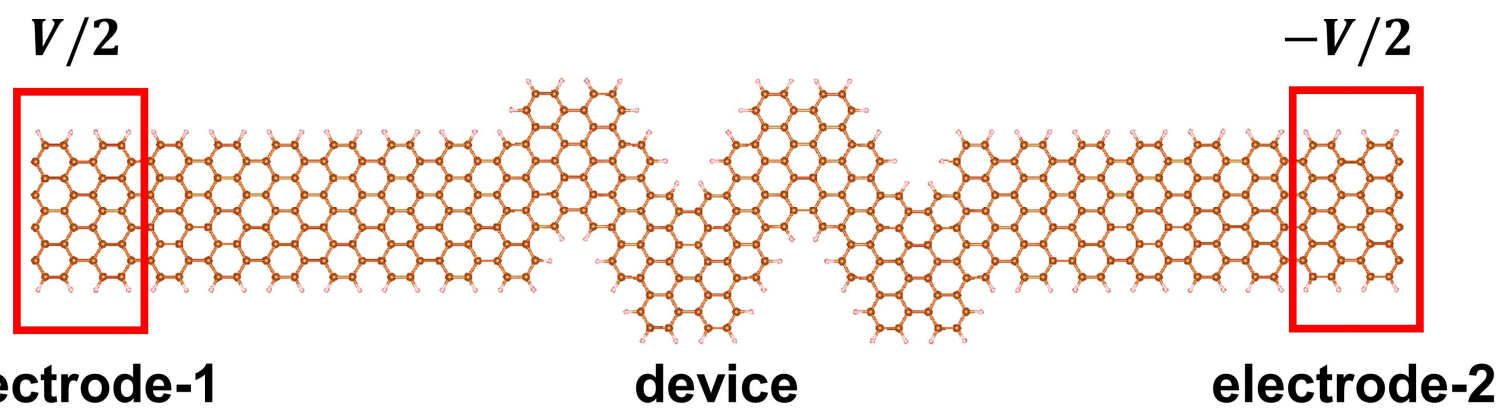

Figure S1 Device setup schematic that is used in performing the NEGF calculations. Electrodes 1 and 2 are the source and drain electrodes, respectively. Both electrodes are 9-armchair GNR to reduce the potential resistance between the electrode and the device regions (e.g., same geometry and material). 


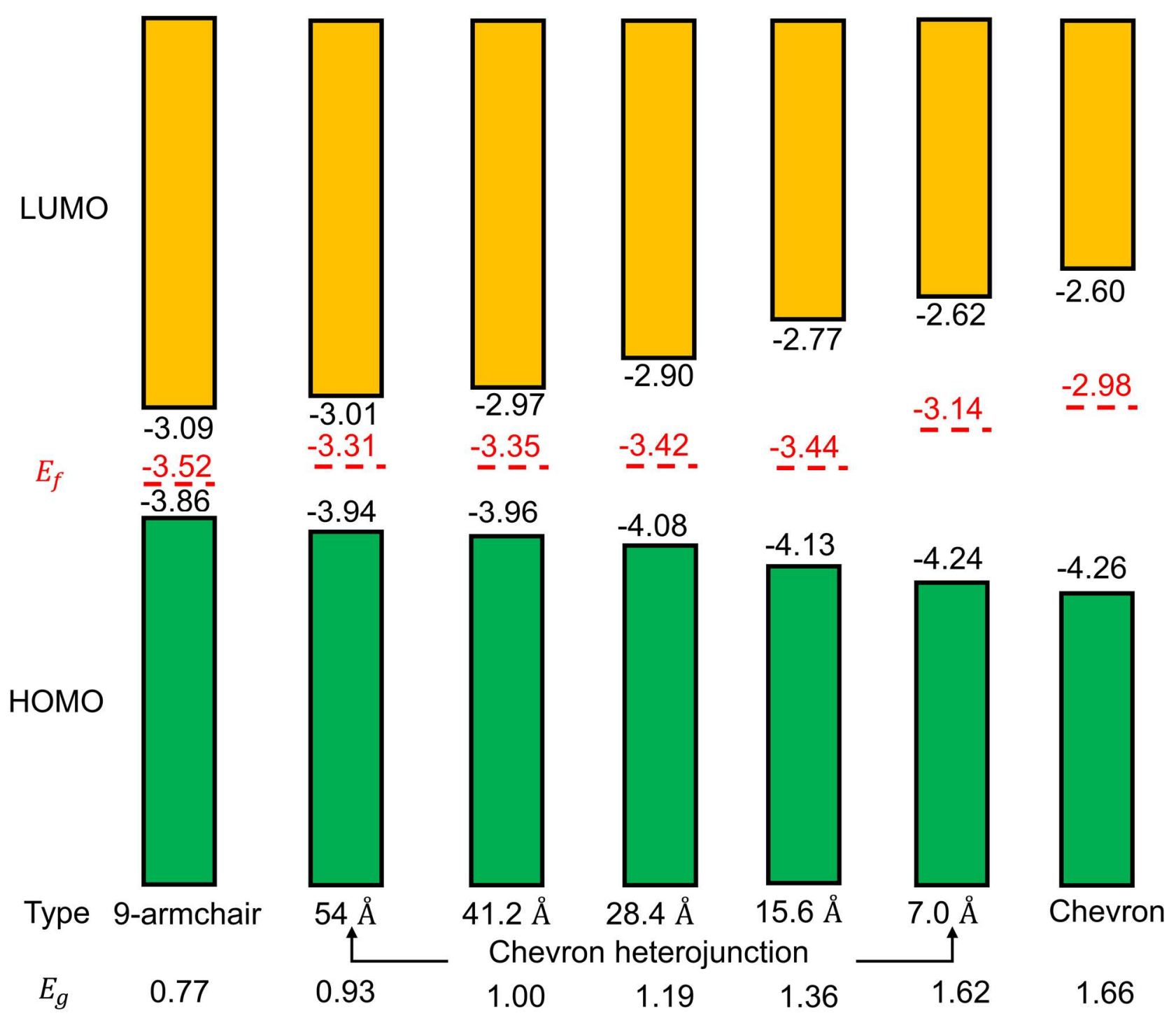

Figure S2 Dependence of the band gap, HOMO, and LUMO energy levels on the lateral length of the 9-armchair GNR for the chevron GNR heterojunctions. 


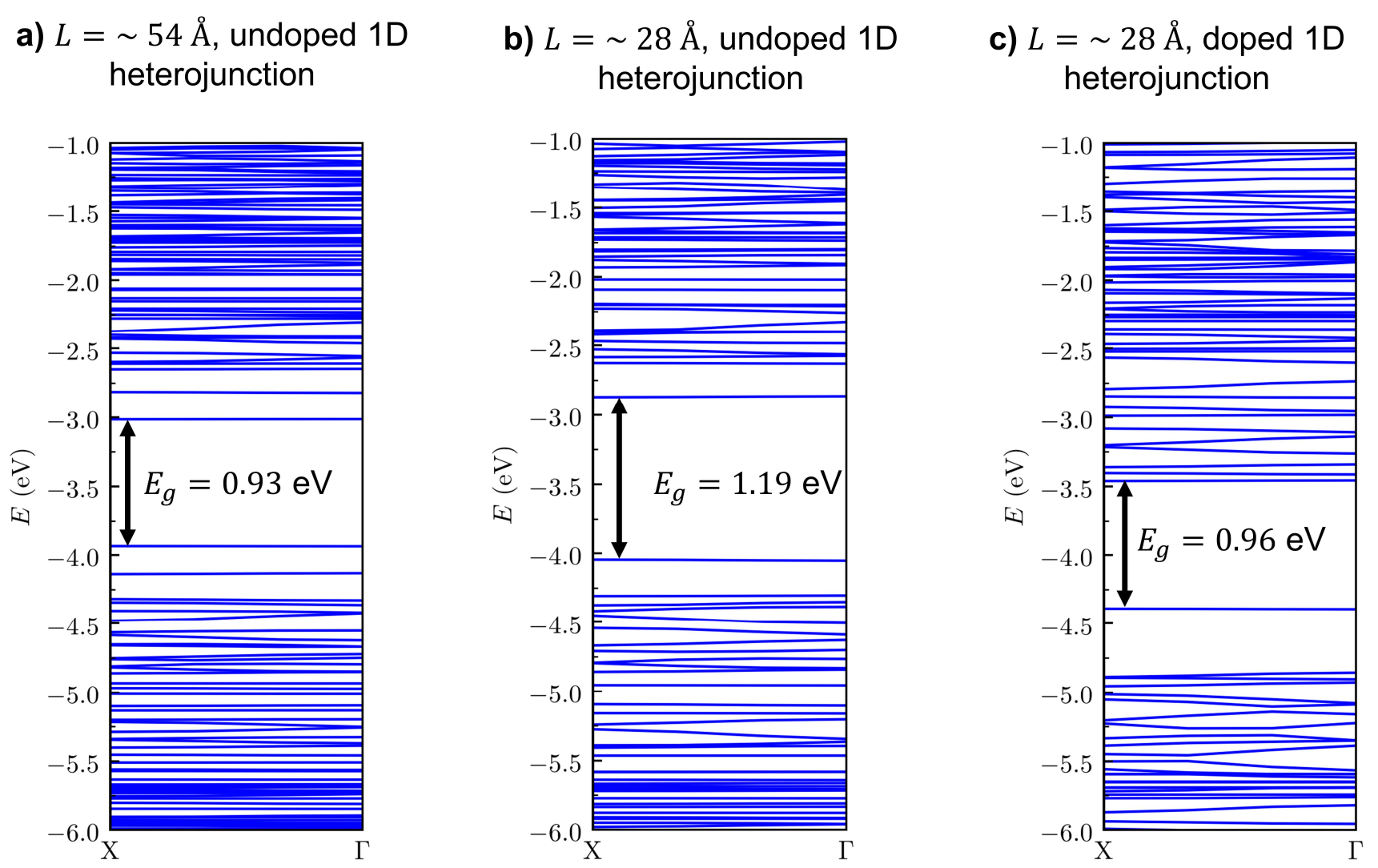

Figure S3 Band structure for a) undoped chevron with $L=54 \AA$, b) undoped chevron with $L=28.4$ $\AA$, c) nitrogen-doped chevron with $L=28.4 \AA$ heterojunctions. 


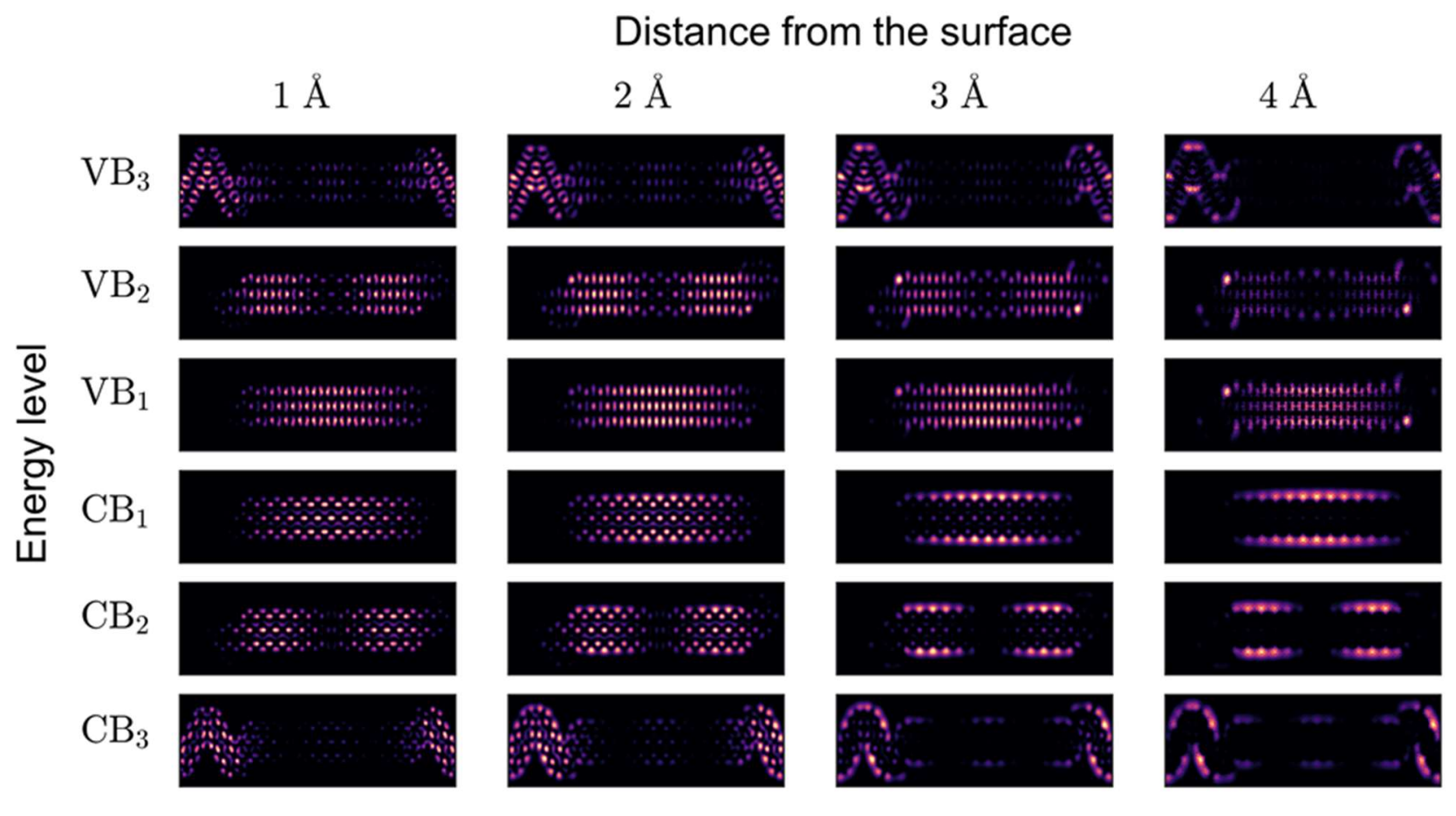

Figure S4 LDOS maps at various energy levels and elevations from the GNR plane for the undoped 1D heterojunction with $L=54 \AA$. The dark regions have no electronic states and the brightest regions have the maximum number of electronic states. 


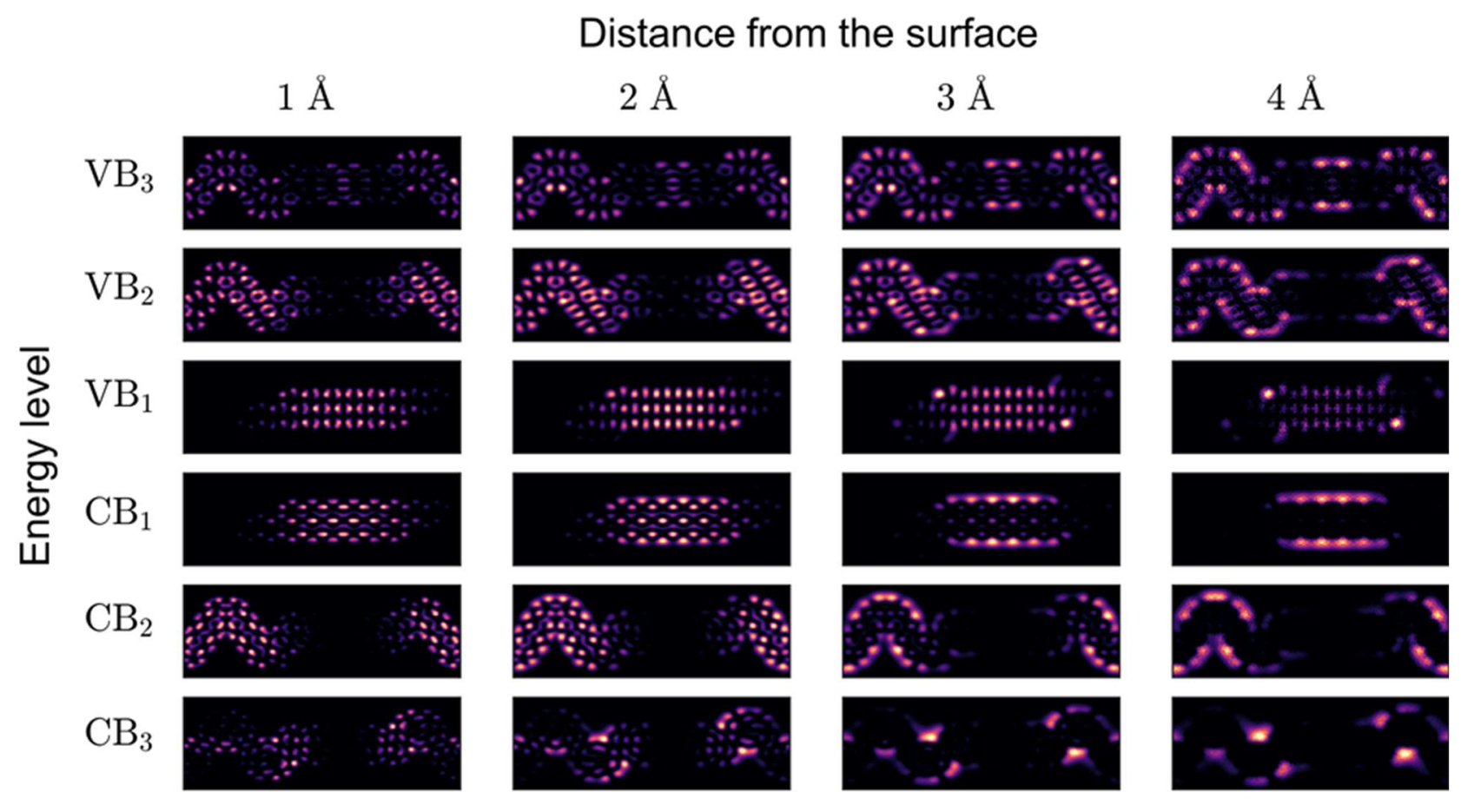

Figure S5 LDOS maps at various energy levels and elevations from the GNR plane for the undoped 1D heterojunction with $L=28.4 \AA$. The dark regions have no electronic states and the brightest regions have the maximum number of electronic states. 
a) $L=\sim 54 \AA$, undoped 1D heterojunction

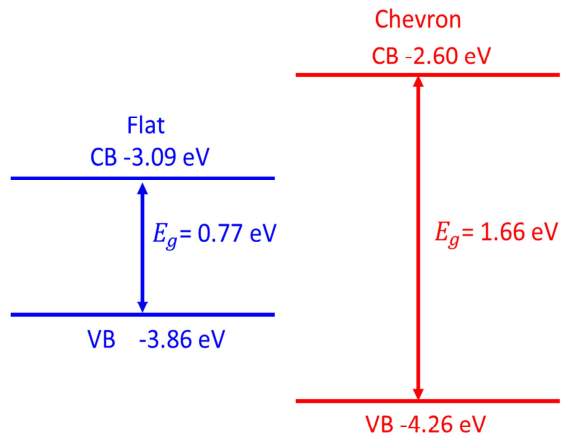

VB -4.26 eV b) $L=\sim 28 \AA$, undoped 1D heterojunction

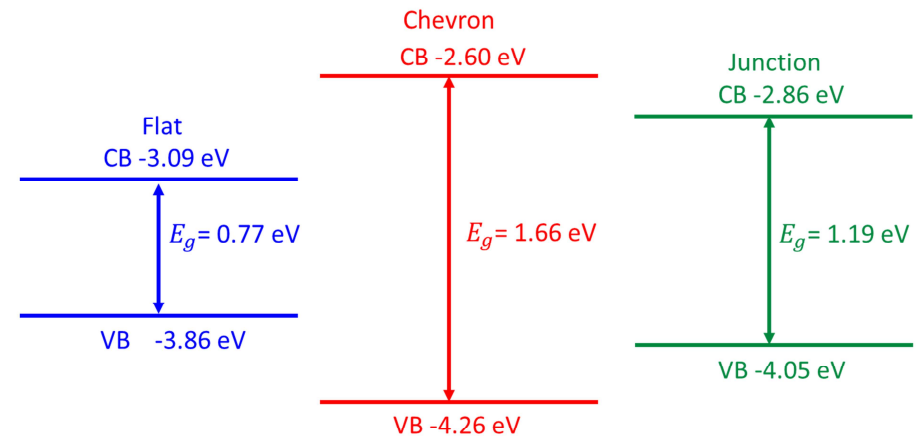

c) $L=\sim 28 \AA$, doped 1D heterojunction
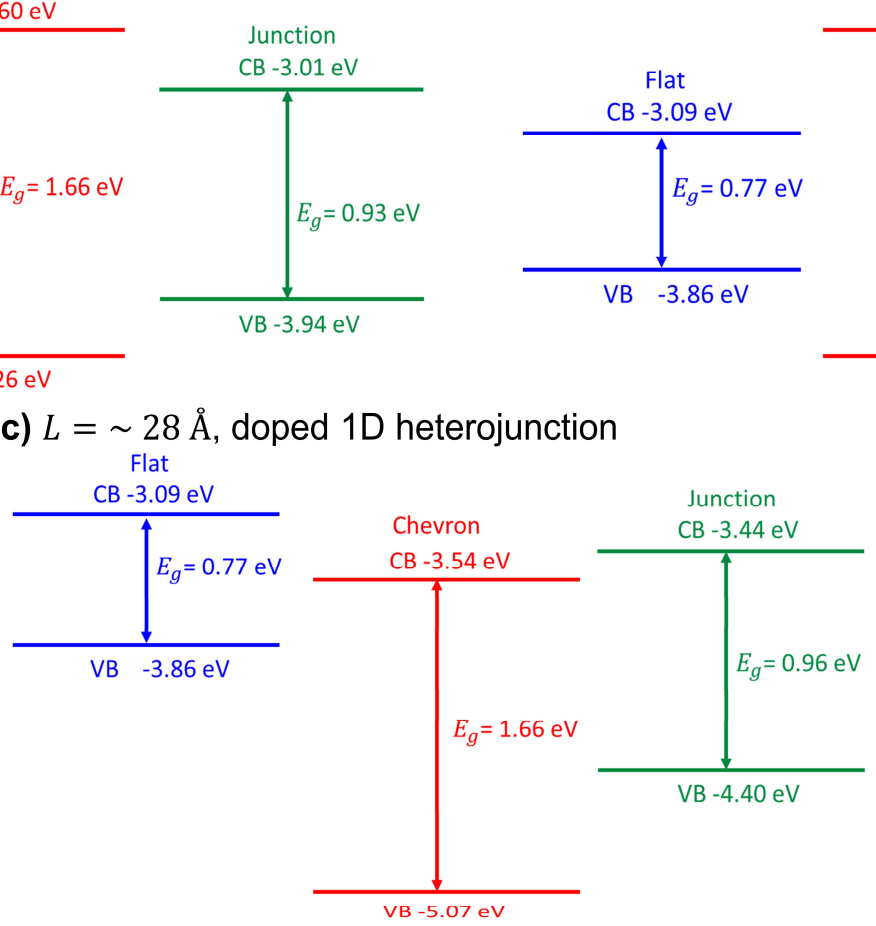

Figure S6 Energy levels of the flat 9-armchair, chevron, and their heterojunction for a) undoped chevron with $L=54 \AA$, b) undoped chevron with $L=28.4 \AA$, c) nitrogen-doped chevron with $L=28.4$ $\AA$. The energy levels of the flat 9 -armchair and chevron GNRs are computed before connecting them to form the heterojunction. 


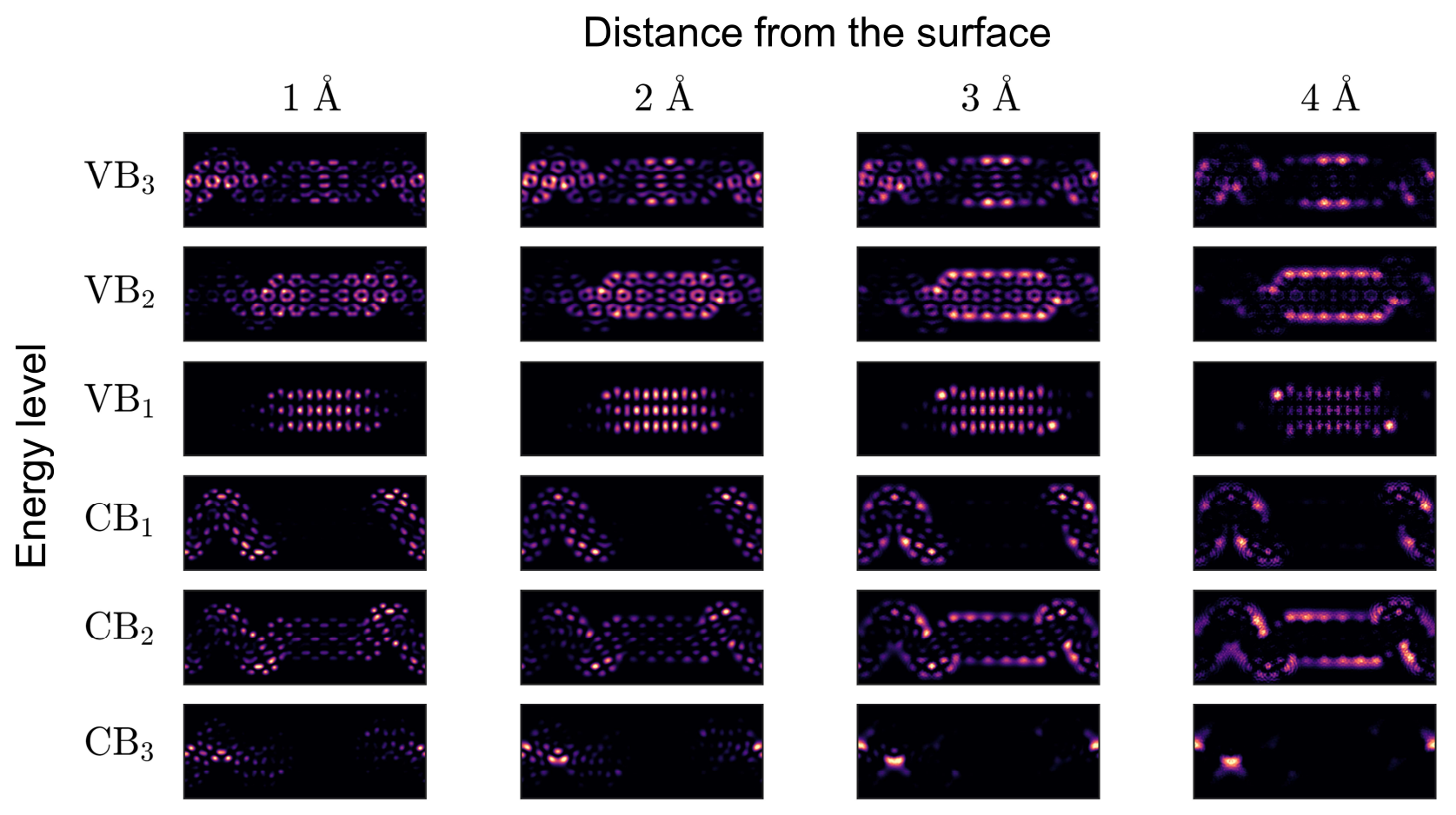

Figure S7 LDOS maps at various energy levels and elevations from the GNR plane for the doped 1D heterojunction with $L=28.4 \AA$. The dark regions have no electronic states and the brightest regions have the maximum number of electronic states. 
a)
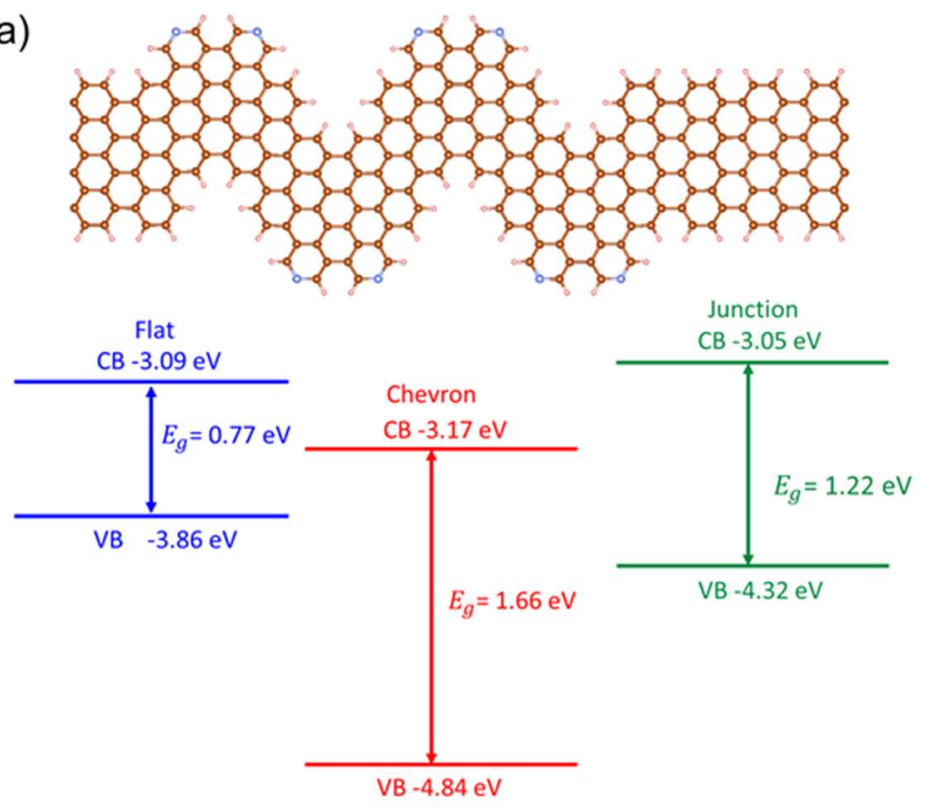

c)

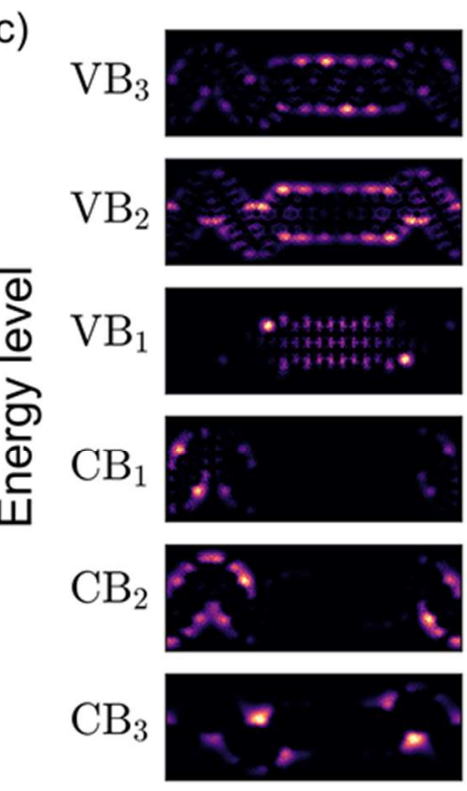

b)
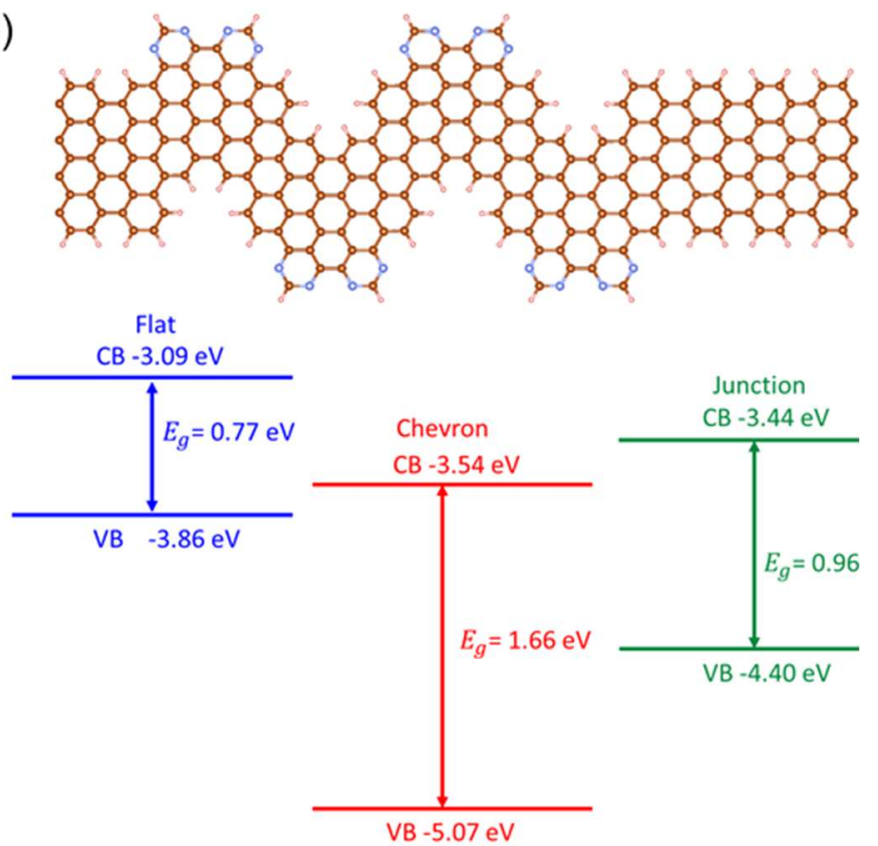

d)

$\mathrm{VB}_{3}$

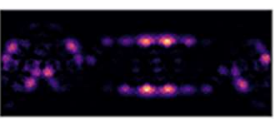

$\mathrm{VB}_{2}$
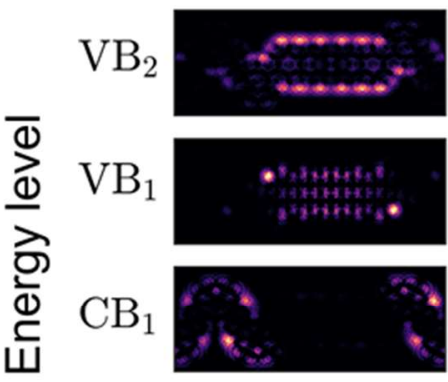

$\mathrm{CB}_{2}$

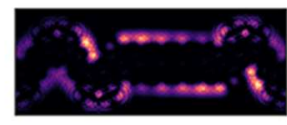

$\mathrm{CB}_{3}$

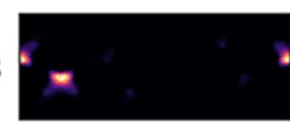

Figure S8 a-b) Energy levels of the flat 9-armchair, chevron, and their heterojunction for 8 and 16 nitrogen atoms doping, respectively, and their corresponding LDOS maps at various energy levels and $4 \AA$ elevation from the GNR plane for $1 \mathrm{D}$ heterojunction with $L=28.4 \AA$. The dark regions have no electronic states and the brightest regions have the maximum number of electronic states. 
a)

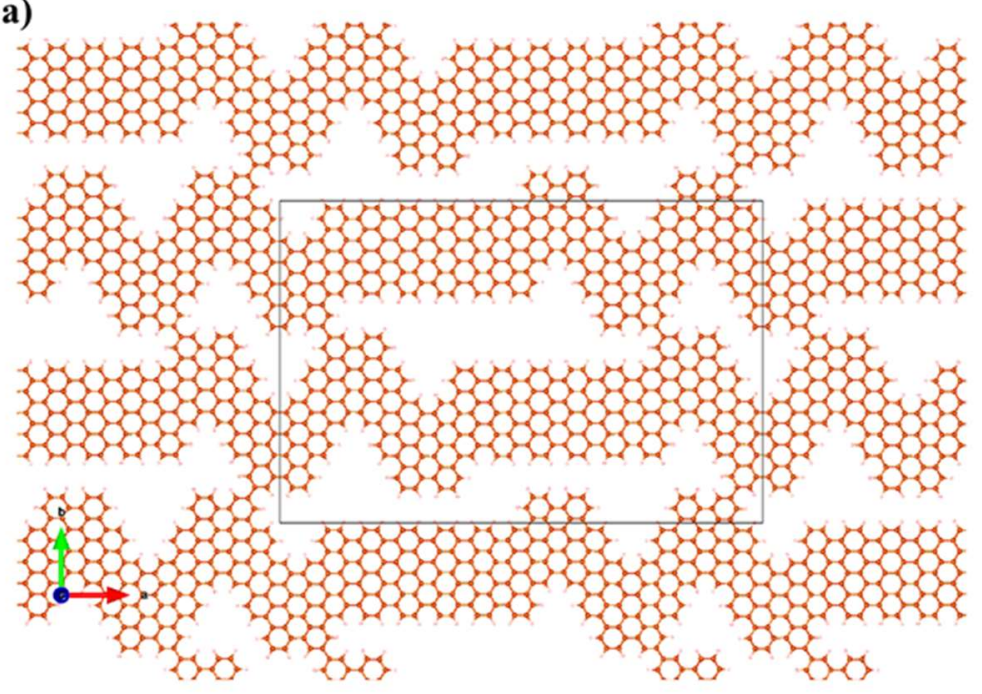

b)

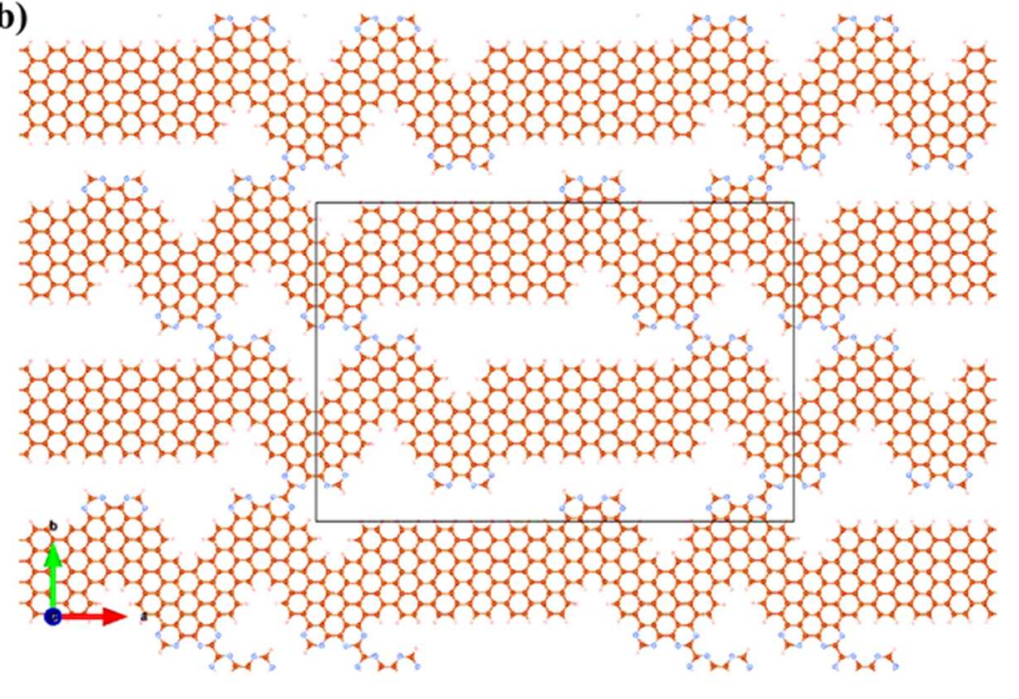

Figure S9 Unit cell of 2D heterojunctions for a) undoped and b) doped chevron GNRs. 


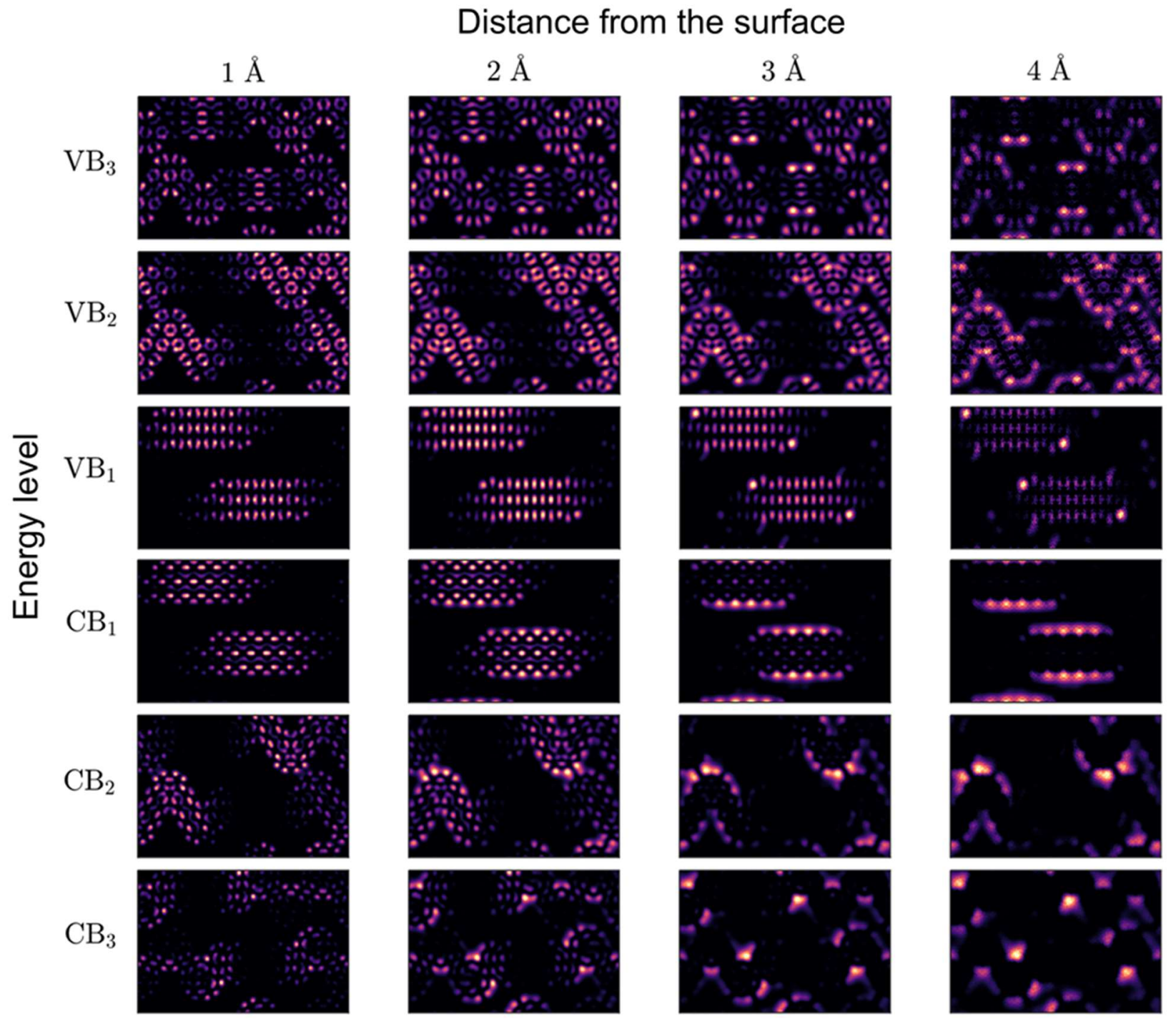

Figure S10 LDOS maps at various energy levels and elevations from the GNR plane for the undoped 2D heterojunction with $L=28.4 \AA$. The dark regions have no electronic states and the brightest regions have the maximum number of electronic states. 


\section{Distance from the surface}

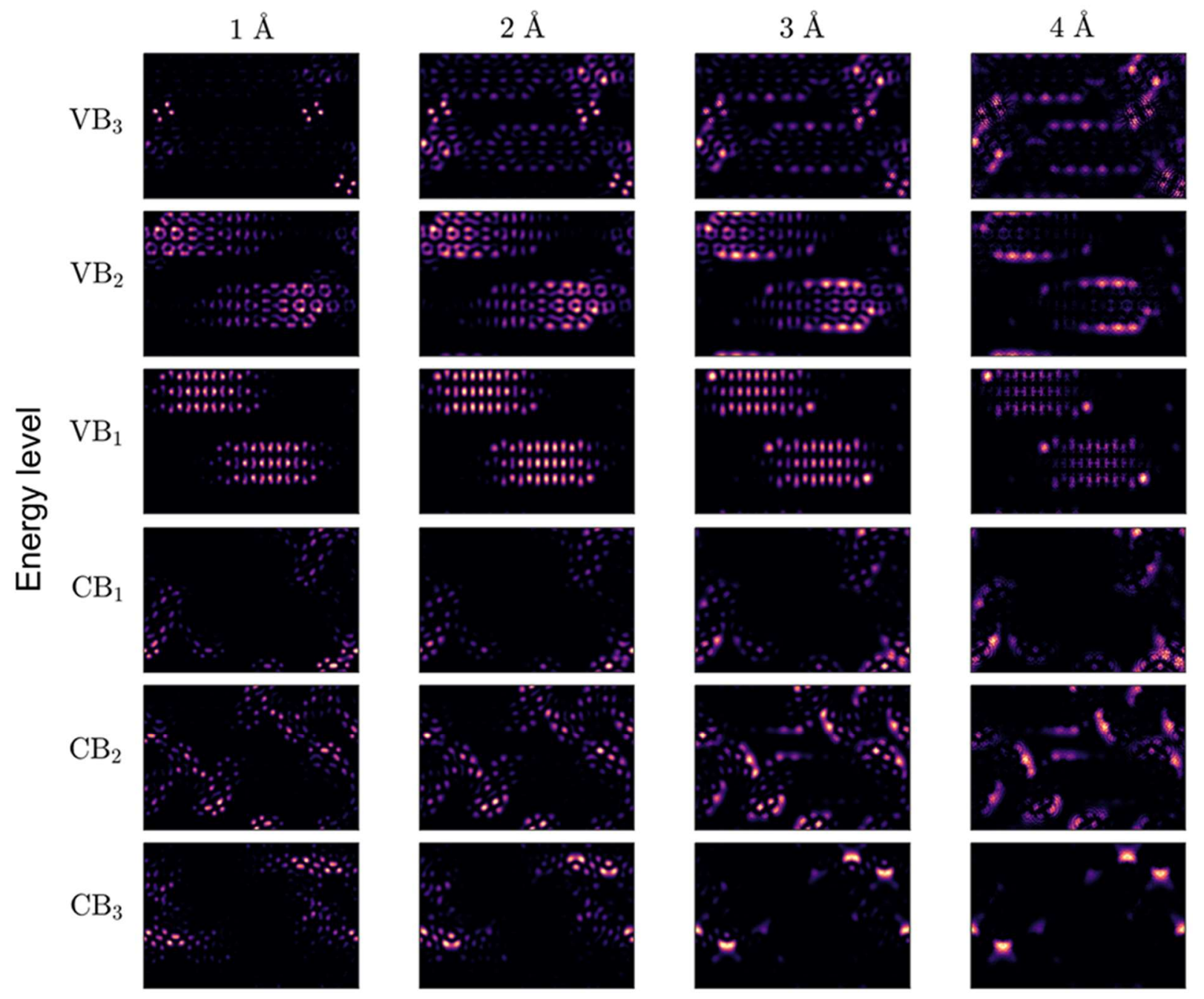

Figure S11 LDOS maps at various energy levels and elevations from the GNR plane for doped 2D heterojunction with $L=28.4 \AA$. The dark regions have no electronic states and the brightest regions have the maximum number of electronic states. 


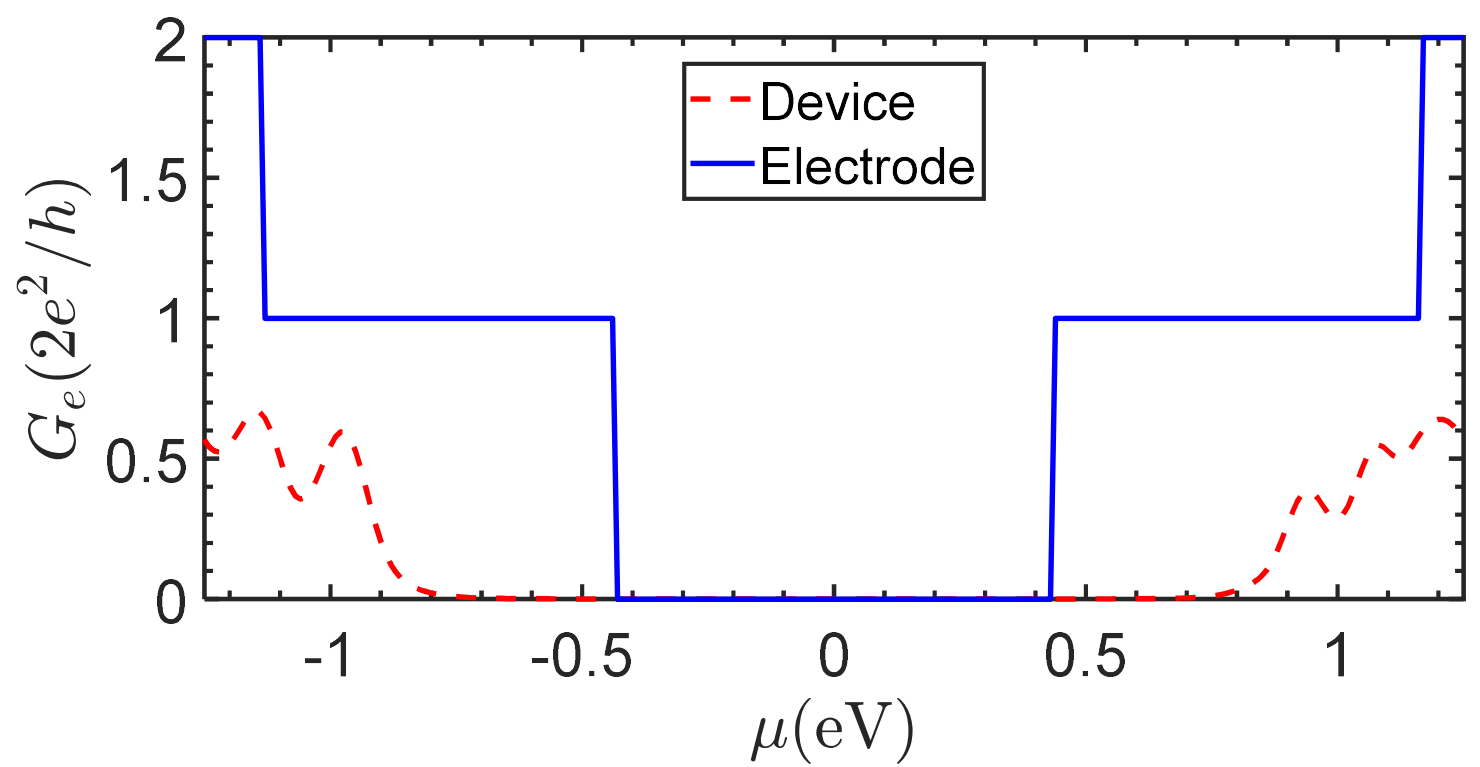

Figure S12 The electronic conductance of the device region (undoped heterojunction) and the quantized electronic conductance of the electrode region (9-armchair GNR) at zero bias as a function of the electrochemical potential. 

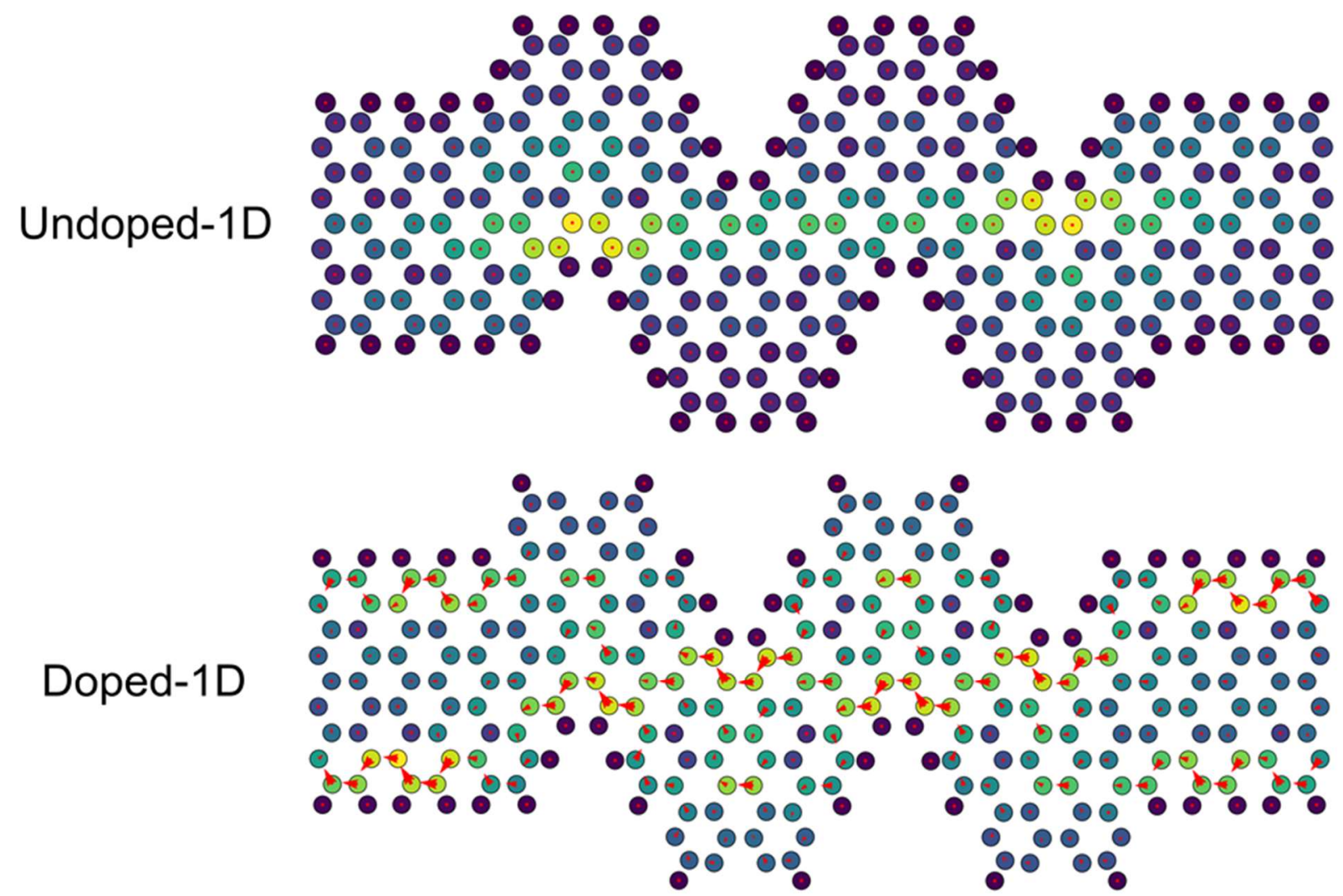

Min

Max

Figure S13 Probability distribution of current with scaled vectors for the different GNR heterojunctions obtained at zero bias and chemical potential corresponds to the first peak in the negative region shown in Figure 4e. 

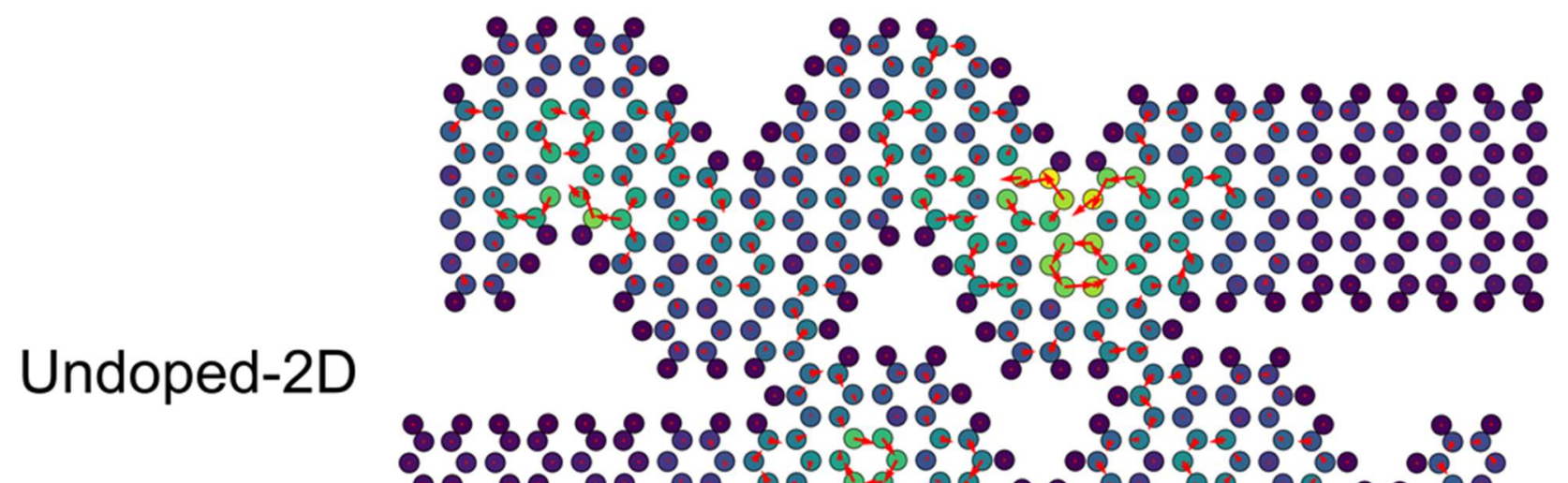

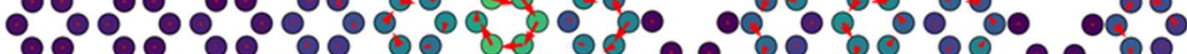

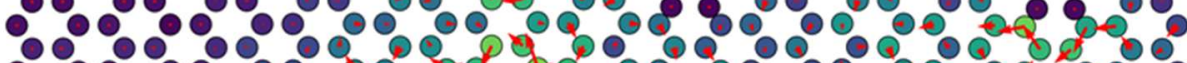

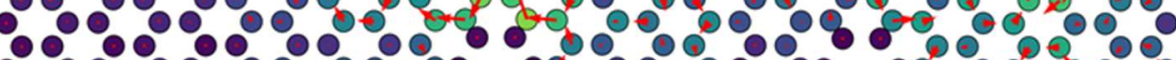

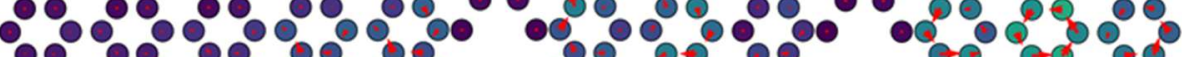
$.888 .88888^{\circ}$ 188

Doped-2D
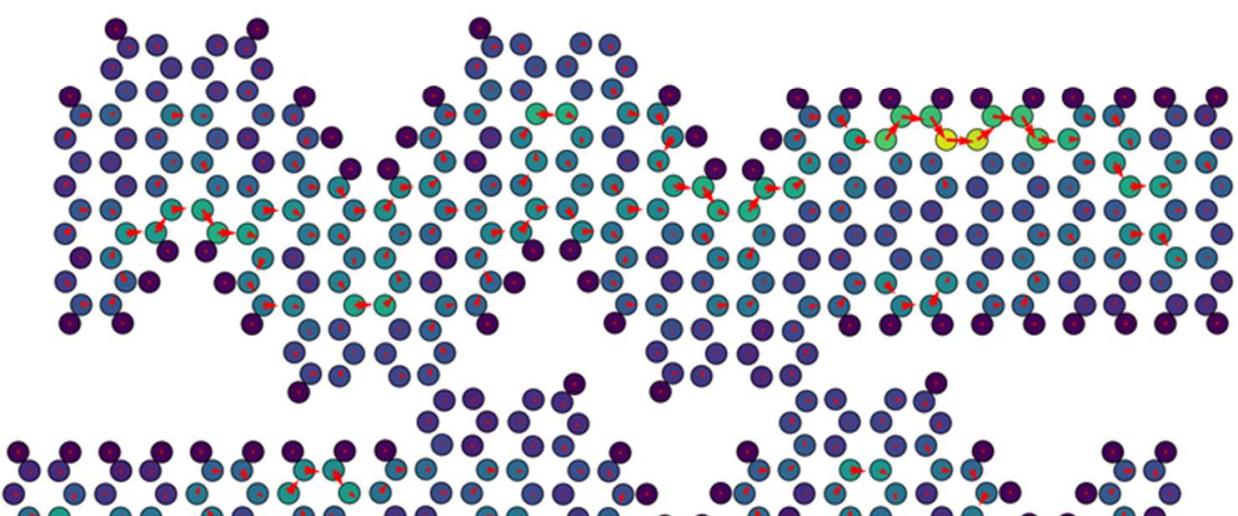

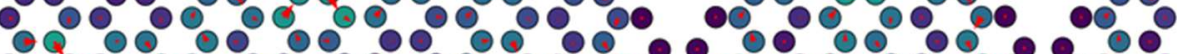

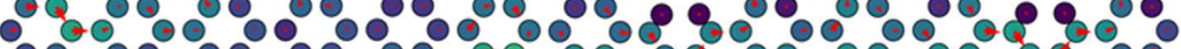

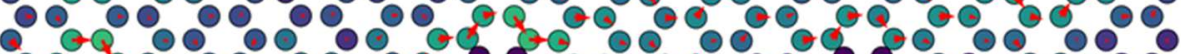

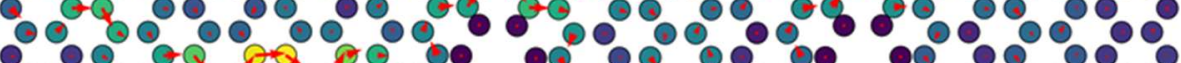

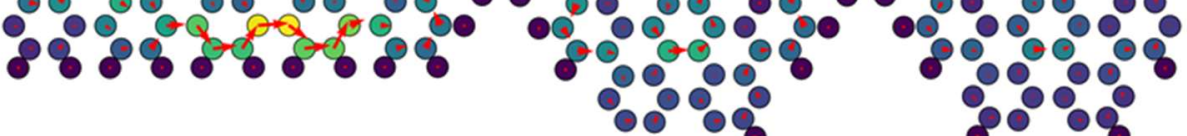
Min

$\operatorname{Max}$

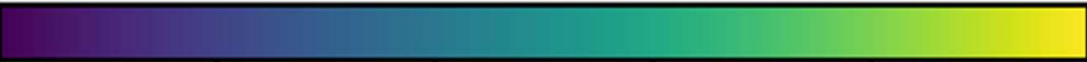

Figure S14 Probability distribution of current with scaled vectors for the different GNR heterojunctions obtained at zero bias and chemical potential corresponds to the first peak in the negative region shown in Figure 4e. 


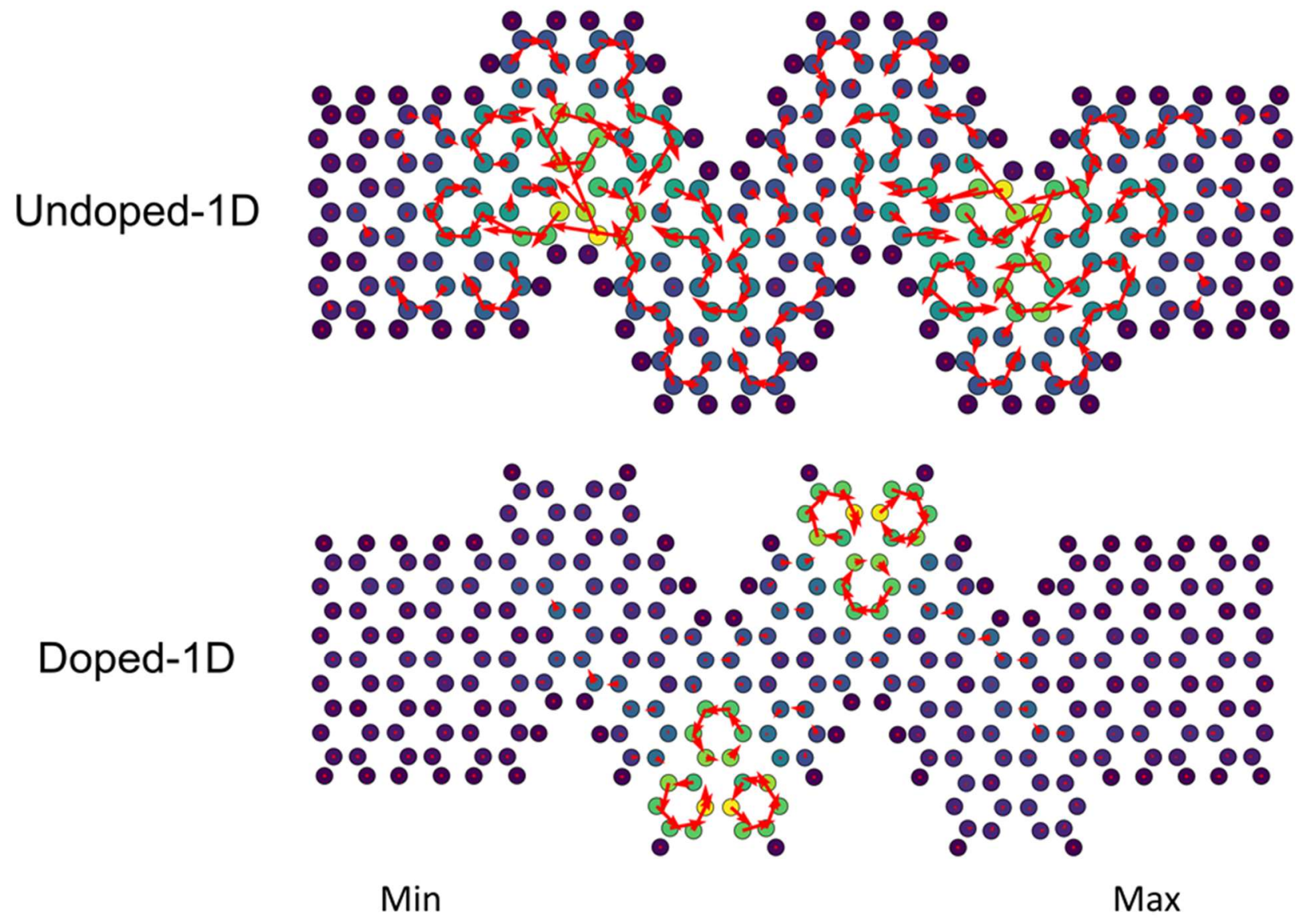

Figure S15 Probability distribution of current with scaled vectors for the different GNR heterojunctions obtained at zero bias and chemical potential corresponds to the first peak in the positive region shown in Figure 4e. 


\section{Undoped-2D}

Doped-2D
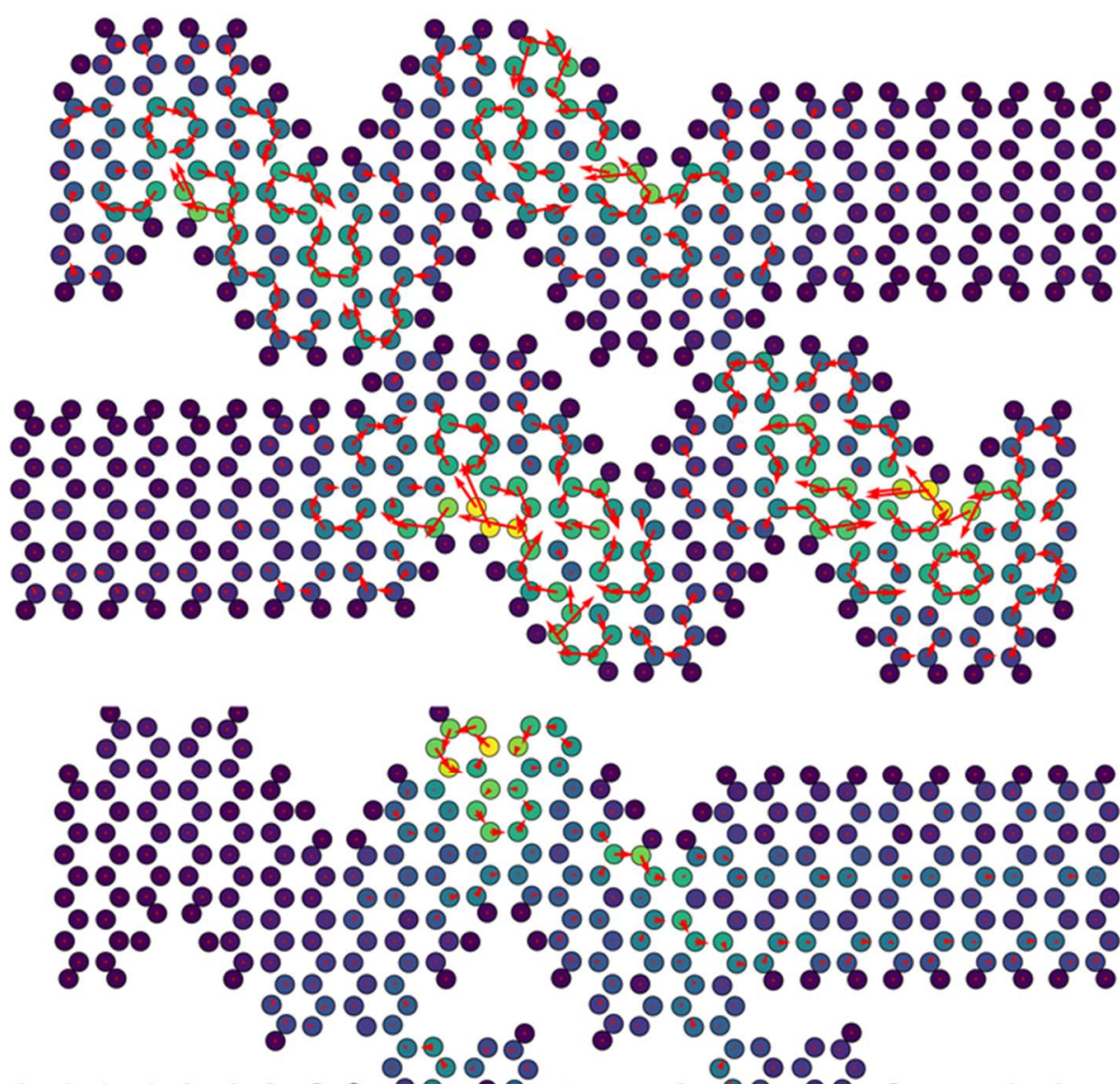

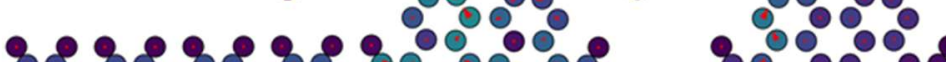

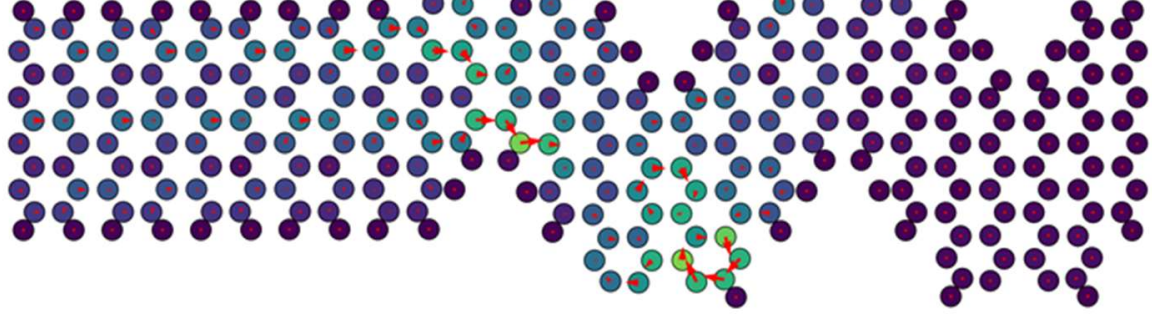

Min

$\operatorname{Max}$

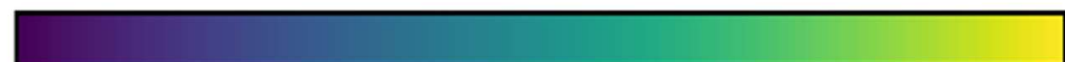

Figure S16 Probability distribution of current with scaled vectors for the different GNR heterojunctions obtained at zero bias and chemical potential corresponds to the first peak in the positive region shown in Figure 4e. 